\title{
Dynamics of entanglement in the transverse Ising model
}

\author{
Zhe Chang*and Ning $\mathrm{Wu}^{\dagger}$ \\ Institute of High Energy Physics \\ Chinese Academy of Sciences \\ P. O. Box 918(4), 100049 Beijing, China
}

\begin{abstract}
We study the evolution of nearest-neighbor entanglement in the one dimensional Ising model with an external transverse field. The system is initialized as the so called "thermal ground state" of the pure Ising model. We analyze properties of generation of entanglement for different regions of external transverse fields. We find that the derivation of the time at which the entanglement reaches its first maximum with respect to the reciprocal transverse field has a minimum at the critical point. This is a new indicator of quantum phase transition.
\end{abstract}

PACS numbers: 03.67.Bg, 75.10.Pq

\section{Introduction}

Entanglement has been recognized as an important resource for quantum information and computation [1], and has been the subject of intense research over the past few years [2]. Recently, there has been extensive analysis of entanglement in quantum spin models [3]. Various models have been considered for entanglement generation, and their static [4, 5] as well as dynamical properties [6, 7, 8] have been investigated. The reason for this is that, many of these models can be realized in cold atomic gas in an optical lattice [9, 10].

A special one among these models is the anisotropic XY model. It can be solved exactly by means of the Jordan-Wigner transformation [11. A lot of works have been done on the dynamics of entanglement in this model. In Ref.[6], the time evolution of initial Bell states was studied. Ref.[7] investigated the dynamics of entanglement of a system prepared in a thermal equilibrium state, and the system starts evolving after the transverse field was turned off.

*e-mail: changz@mail.ihep.ac.cn

${ }^{\dagger}$ e-mail: wun@mail.ihep.ac.cn 
In this paper, we analyze a spin- $1 / 2$ transverse Ising chain with a particular separable initial state, the so-called "thermal ground state" [4] for the case of zero field. We study the generation of nearest-neighbor entanglement as a function of the external transverse field, and find the time at which the entanglement reaches its first maximum. It turns out that the derivation of this time with respect to the reciprocal transverse field has a minimum at the critical point. This is a new indicator of quantum phase transition.

\section{Static correlations}

The anisotropic XY model is described by the Hamiltonian

$$
H=-\frac{\lambda}{4} \sum_{i=1}^{N}\left[(1+\gamma) \sigma_{i}^{x} \sigma_{i+1}^{x}+(1-\gamma) \sigma_{i}^{y} \sigma_{i+1}^{y}\right]-\frac{1}{2} \sum_{i=1}^{N} \sigma_{i}^{z},
$$

where $\sigma_{i}^{\alpha}$ are the Pauli matrix of the spin at site $i$ and we assume periodic boundary conditions. We have neglected a common factor and absorbed the external field into the reciprocal field $\lambda$. The anisotropy parameter $\gamma$ connects the quantum Ising model for $\gamma=1$. In the range $0<\gamma \leq 1$, the model belongs to the Ising universality class and undergoes a quantum phase transition at the critical point $\lambda_{c}=1$. We will consider the case of $\gamma=1$ in this paper, namely the transverse Ising model

$$
H=-\frac{1}{2} \sum_{i=1}^{N}\left(\lambda \sigma_{i}^{x} \sigma_{i+1}^{x}+\sigma_{i}^{z}\right) .
$$

The Hamiltonian defined in Eq.(2) can be mapped into a one-dimensional spinless fermion system with creation and annihilation operators $c_{i}^{\dagger}$ and $c_{i}$ via the Jordan-Wigner transformation $\frac{\sigma_{i}^{x}-i \sigma_{i}^{y}}{2}=\prod_{j<i}\left(1-2 c_{j}^{\dagger} c_{j}\right) c_{i}, \frac{\sigma_{i}^{x}+i \sigma_{i}^{y}}{2}=c_{i}^{\dagger} \prod_{j<i}\left(1-2 c_{j}^{\dagger} c_{j}\right), \frac{\sigma_{i}^{z}}{2}=c_{i}^{\dagger} c_{i}-\frac{1}{2}$. By making use of the transformation

$$
\eta_{k}=\frac{1}{\sqrt{N}} \sum_{l} e^{i k l}\left(\alpha_{k} c_{l}+i \beta_{k} c_{l}^{\dagger}\right)
$$

further, with $\alpha_{k}=\frac{\Lambda_{k}-(1+\lambda \cos k)}{\sqrt{2\left[\Lambda_{k}^{2}-(1+\lambda \cos k) \Lambda_{k}\right]}}, \quad \beta_{k}=\frac{\lambda \sin k}{\sqrt{2\left[\Lambda_{k}^{2}-(1+\lambda \cos k) \Lambda_{k}\right]}}$, we get the fermionic Hamiltonian finally

$$
H=\sum_{k} \Lambda_{k}\left(\eta_{k}^{\dagger} \eta_{k}-\frac{1}{2}\right)
$$

where the spectrum is $\Lambda_{k}=\sqrt{1+\lambda^{2}+2 \lambda \cos k}$.

The evolution of the system is governed by Eq.(2). In the Heisenberg picture, the time evolution of the spinless fermion operator $c_{i}(t)$ is [6]

$$
c_{i}(t)=\frac{1}{N} \sum_{k, l}\left[\cos k(l-i) A(k, t) c_{l}+\sin k(l-i) B(k, t) c_{l}^{\dagger}\right] \text {, }
$$


where $A(k, t)=e^{i \Lambda_{k} t}-2 i \beta_{k}^{2} \sin \Lambda_{k} t, B(k, t)=-2 i \alpha_{k} \beta_{k} \sin \Lambda_{k} t$.

The dynamics of the system also depends on the initial state. We choose the "thermal ground state" $\rho_{0}=\frac{1}{2}\left(\left|N^{+}\right\rangle\left\langle N^{+}|+| N^{-}\right\rangle\left\langle N^{-}\right|\right)$of the pure Ising model (no transverse field is applied) as the initial state. Here $\left|N^{+}\right\rangle=|\rightarrow\rangle_{1} \ldots|\rightarrow\rangle_{N}$, and $\left|N^{-}\right\rangle=|\leftarrow\rangle_{1} \ldots|\leftarrow\rangle_{N}$ are the two degenerate ground states of the Ising model with all spins pointing to the positive (negative) $x$ direction. Note that $\left|N^{+}\right\rangle$and $\left|N^{-}\right\rangle$are the ground states of the Hamiltonian (2) with $\lambda \rightarrow \infty$. When $\lambda$ is finite, they are even not eigenstates of the Hamiltonian. Thus, the evolution from this initial state must be nontrivial for finite $\lambda$. Physically, this can be viewed as an instantaneous, i.e., idealized sudden quench [8] in the reciprocal field $\lambda_{1} \rightarrow \lambda_{2}$ with $\lambda_{1} \rightarrow \infty$ and $\lambda_{2}=\lambda$.

Due to the translational symmetry, we need only to consider averages of the form $\left\langle c_{1} \ldots\right\rangle_{0}$. Namely, we choose site-1 as "the first site", where $\langle\ldots\rangle_{0}$ denote the average over the initial state $\rho_{0}$. We rewrite $\left|N^{ \pm}\right\rangle$in the fermionic representation,

$$
\left|N^{ \pm}\right\rangle=\left(\frac{1}{\sqrt{2}}\right)^{N}\left(1 \pm c_{1}^{\dagger}\right)\left(1 \pm c_{2}^{\dagger}\right) \ldots\left(1 \pm c_{N}^{\dagger}\right)\left|0_{1}, 0_{2}, \ldots, 0_{N}\right\rangle,
$$

where $\left|0_{1}, 0_{2}, \ldots, 0_{N}\right\rangle$ denotes the vacuum of the fermions, or the state for all spins down. Here we have to pay attention to the order of operators in this equation. We write $\left(1 \pm c_{1}^{\dagger}\right)$ before operators on all other sites in order to be compatible with the correlation $\left\langle c_{1} \ldots\right\rangle_{0}$. In other words, if another site $i$ is chosen as "the first site", we should write the state as $\left|N^{ \pm}\right\rangle=\left(\frac{1}{\sqrt{2}}\right)^{N}\left(1 \pm c_{i}^{\dagger}\right)\left(1 \pm c_{i+1}^{\dagger}\right) \ldots\left(1 \pm c_{i-1}^{\dagger}\right)\left|0_{i}, 0_{i+1}, \ldots, 0_{i-1}\right\rangle$ to calculate averages like $\left\langle c_{i} \ldots\right\rangle_{0}$, due to the periodic boundary conditions.

The only single-site averages are $\left\langle c_{1}\right\rangle_{0}$ and $\left\langle c_{1}^{\dagger} c_{1}\right\rangle_{0}$ (or their complex conjugate). It is easy to see that $\left\langle N^{+}\left|c_{1}\right| N^{+}\right\rangle=\frac{1}{2}=-\left\langle N^{-}\left|c_{1}\right| N^{-}\right\rangle$, so $\left\langle c_{1}\right\rangle_{0}=0$. As to $\left\langle c_{1}^{\dagger} c_{1}\right\rangle_{0}$, we have $\left\langle N^{+}\left|c_{1}^{\dagger} c_{1}\right| N^{+}\right\rangle=\frac{1}{2}\left\langle 0_{1}\left|\left(1+c_{1}\right) c_{1}^{\dagger} c_{1}\left(1+c_{1}^{\dagger}\right)\right| 0_{1}\right\rangle=\frac{1}{2}$. Similarly, $\left\langle N^{-}\left|c_{1}^{\dagger} c_{1}\right| N^{-}\right\rangle=\frac{1}{2}$. So that, $\left\langle c_{1}^{\dagger} c_{1}\right\rangle_{0}=\frac{1}{2}$. We can also see this point from $0=\left\langle\frac{\sigma_{1}^{z}}{2}\right\rangle_{0}=\left\langle c_{1}^{\dagger} c_{1}-\frac{1}{2}\right\rangle_{0}$.

Next we consider two-point correlations of the form $\left\langle c_{1} c_{l}\right\rangle_{0}, l \geq 2$. For $l=2$, we have

$$
\begin{aligned}
\left\langle N^{+}\left|c_{1} c_{2}\right| N^{+}\right\rangle & =\left(\frac{1}{2}\right)^{2}\left\langle 0_{1}, 0_{2}\left|\left(1+c_{2}\right)\left(1+c_{1}\right) c_{1} c_{2}\left(1+c_{1}^{\dagger}\right)\left(1+c_{2}^{\dagger}\right)\right| 0_{1}, 0_{2}\right\rangle \\
& =\left(\frac{1}{2}\right)^{2}\left\langle 0_{1}\left|\left(1+c_{1}\right) c_{1}\left(1-c_{1}^{\dagger}\right)\right| 0_{1}\right\rangle \cdot\left\langle 0_{2}\left|\left(1+c_{2}\right) c_{2}\left(1+c_{2}^{\dagger}\right)\right| 0_{2}\right\rangle \\
& =-\frac{1}{4} .
\end{aligned}
$$

Similarly $\left\langle N^{-}\left|c_{1} c_{2}\right| N^{-}\right\rangle=-\frac{1}{4}$. So that $\left\langle c_{1} c_{2}\right\rangle_{0}=-\frac{1}{4}$. We can show that $\left\langle c_{1}^{\dagger} c_{2}\right\rangle_{0}=\frac{1}{4}$ in the same manner. For $l>2$, when $c_{l}$ or $c_{l}^{\dagger}$ crosses $\left(1 \pm c_{2}^{\dagger}\right)$ to its right side, this will make it be $\left(1 \mp c_{2}^{\dagger}\right)$. But $\left\langle 0_{2}\left|\left(1 \pm c_{2}\right)\left(1 \mp c_{2}^{\dagger}\right)\right| 0_{2}\right\rangle=0$. So we have obtained all the two-point correlations

$$
\begin{aligned}
\left\langle c_{i}^{\dagger} c_{j}\right\rangle_{0} & =\frac{1}{4}\left(\delta_{i+1, j}+\delta_{i-1, j}+2 \delta_{i, j}\right), \\
\left\langle c_{i} c_{j}\right\rangle_{0} & =\frac{1}{4}\left(-\delta_{i+1, j}+\delta_{i-1, j}\right), \\
\left\langle c_{i}^{\dagger} c_{j}^{\dagger}\right\rangle_{0} & =\frac{1}{4}\left(\delta_{i+1, j}-\delta_{i-1, j}\right) .
\end{aligned}
$$


By the same means one can check that the averages involving three fermion operators all vanish.

\section{Reduced density matrix and evolution of concur- rence}

Making use of the periodic boundary conditions, we can focus on the reduced density matrix $\rho^{12}(t)$ at time $t$ of the first and second spins. In the $\{|\uparrow \uparrow\rangle,|\uparrow \downarrow\rangle,|\downarrow \uparrow\rangle,|\downarrow \downarrow\rangle\}$ basis, the matrix elements reads,

$$
\begin{aligned}
\rho_{11}^{12}(t)= & \left\langle\uparrow \uparrow\left|\rho^{12}(t)\right| \uparrow \uparrow\right\rangle \\
= & \left\langle\uparrow \uparrow\left|\rho^{12}(t) S_{1}^{+} S_{1}^{-} S_{2}^{+} S_{2}^{-}\right| \uparrow \uparrow\right\rangle+\left\langle\uparrow \downarrow\left|\rho^{12}(t) S_{1}^{+} S_{1}^{-} S_{2}^{+} S_{2}^{-}\right| \uparrow \downarrow\right\rangle \\
& +\left\langle\downarrow \uparrow\left|\rho^{12}(t) S_{1}^{+} S_{1}^{-} S_{2}^{+} S_{2}^{-}\right| \downarrow \uparrow\right\rangle+\left\langle\downarrow \downarrow\left|\rho^{12}(t) S_{1}^{+} S_{1}^{-} S_{2}^{+} S_{2}^{-}\right| \downarrow \downarrow\right\rangle \\
= & \operatorname{tr}_{12}\left(\rho^{12}(t) S_{1}^{+} S_{1}^{-} S_{2}^{+} S_{2}^{-}\right) \\
= & \operatorname{tr}\left(\rho(t) S_{1}^{+} S_{1}^{-} S_{2}^{+} S_{2}^{-}\right) \\
= & \left\langle c_{1}^{\dagger} c_{1} c_{2}^{\dagger} c_{2}\right\rangle_{t} .
\end{aligned}
$$

Other matrix elements can be obtained similarly. Thus, we have

$$
\rho^{12}(t)=\left(\begin{array}{cccc}
\left\langle c_{1}^{\dagger} c_{1} c_{2}^{\dagger} c_{2}\right\rangle_{t} & -\left\langle c_{1}^{\dagger} c_{1} c_{2}\right\rangle_{t} & \left\langle c_{2}^{\dagger} c_{2} c_{1}\right\rangle_{t} & \left\langle c_{2} c_{1}\right\rangle_{t} \\
-\left\langle c_{2}^{\dagger} c_{1}^{\dagger} c_{1}\right\rangle_{t} & \left\langle c_{1}^{\dagger} c_{1} c_{2} c_{2}^{\dagger}\right\rangle_{t} & -\left\langle c_{1} c_{2}^{\dagger}\right\rangle_{t} & \left\langle c_{1} c_{2} c_{2}^{\dagger}\right\rangle_{t} \\
\left\langle c_{1}^{\dagger} c_{2}^{\dagger} c_{2}\right\rangle_{t} & -\left\langle c_{2} c_{1}^{\dagger}\right\rangle_{t} & \left\langle c_{1} c_{1}^{\dagger} c_{2}^{\dagger} c_{2}\right\rangle_{t} & \left\langle c_{1} c_{1}^{\dagger} c_{2}\right\rangle_{t} \\
\left\langle c_{1}^{\dagger} c_{2}^{\dagger}\right\rangle_{t} & \left\langle c_{2} c_{2}^{\dagger} c_{1}^{\dagger}\right\rangle_{t} & \left\langle c_{2}^{\dagger} c_{1} c_{1}^{\dagger}\right\rangle_{t} & \left\langle c_{1} c_{1}^{\dagger} c_{2} c_{2}^{\dagger}\right\rangle_{t}
\end{array}\right),
$$

All the elements can be equally evaluated in the Heisenberg picture, e.g., $\rho_{11}^{12}(t)=$ $\left\langle c_{1}^{\dagger} c_{1} c_{2}^{\dagger} c_{2}\right\rangle_{t}=\left\langle c_{1}^{\dagger}(t) c_{1}(t) c_{2}^{\dagger}(t) c_{2}(t)\right\rangle_{0}$. We have mentioned that all the static averages involving three fermion operators equal zero. From Eq.(5), the fermion operators in the Heisenberg picture are merely some linear superpositions of the same set of operators in the Schrodinger picture. So all matrix elements involving three operators in Eq.(8) also vanish. Using Wick's theorem, one gets all non-vanishing elements of $\rho^{12}(t)$ as following

$$
\begin{aligned}
& \rho_{11}^{12}(t)=\left\langle c_{1}^{\dagger}(t) c_{1}(t)\right\rangle_{0}\left\langle c_{2}^{\dagger}(t) c_{2}(t)\right\rangle_{0}-\left\langle c_{1}^{\dagger}(t) c_{2}^{\dagger}(t)\right\rangle_{0}\left\langle c_{1}(t) c_{2}(t)\right\rangle_{0}-\left\langle c_{1}^{\dagger}(t) c_{2}(t)\right\rangle\left\langle c_{2}^{\dagger}(t) c_{1}(t)\right\rangle_{0} \\
& \rho_{22}^{12}(t)=\rho_{33}^{12}(t)=\left\langle c_{1}^{\dagger}(t) c_{1}(t)\right\rangle_{0}-\rho_{11}^{12}(t) \\
& \rho_{44}^{12}(t)=1-\rho_{11}^{12}(t)-2 \rho_{22}^{12}(t) \\
& \rho_{14}^{12}(t)=\rho_{41}^{12 *}(t)=\left\langle c_{2}(t) c_{1}(t)\right\rangle_{0} \\
& \rho_{23}^{12}(t)=\rho_{32}^{12 *}(t)=-\left\langle c_{1}(t) c_{2}^{\dagger}(t)\right\rangle_{0} .
\end{aligned}
$$


We see that if we can calculate $\left\langle c_{1}^{\dagger}(t) c_{1}(t)\right\rangle_{0},\left\langle c_{1}^{\dagger}(t) c_{2}(t)\right\rangle_{0}$ and $\left\langle c_{1}(t) c_{2}(t)\right\rangle_{0}$, then the reduced density matrix is totally determined.

Using Eq.(5) and (7), we find, in the thermodynamic limit, that

$$
\begin{aligned}
\left\langle c_{1}^{\dagger}(t) c_{1}(t)\right\rangle= & \frac{1}{8 \pi} \int_{-\pi}^{\pi} d k \sin k\left(A^{*}(k)-A(k)\right)\left(B(k)-B^{*}(k)\right)+\frac{1}{4 \pi} \int_{-\pi}^{\pi} d k\left(|A(k)|^{2}+|B(k)|^{2}\right) \\
& +\frac{1}{4 \pi} \int_{-\pi}^{\pi} d k \cos k\left(|A(k)|^{2}-|B(k)|^{2}\right) \\
= & \frac{1}{\pi} \int_{0}^{\pi} d k \frac{\lambda \sin ^{2} k \sin ^{2} \Lambda_{k} t}{\Lambda_{k}^{2}}+\frac{1}{2}, \\
\left\langle c_{1}^{\dagger}(t) c_{2}(t)\right\rangle= & \frac{1}{8 \pi} \int_{-\pi}^{\pi} d k(1+\cos 2 k)\left(|A(k)|^{2}-|B(k)|^{2}\right)+\frac{1}{4 \pi} \int_{-\pi}^{\pi} d k \cos k\left(|A(k)|^{2}+|B(k)|^{2}\right) \\
& +\frac{1}{8 \pi} \int_{-\pi}^{\pi} d k \sin 2 k\left[A(k)-A^{*}(k)\right] B^{*}(k) \\
= & \frac{1}{\pi} \int_{0}^{\pi} d k \frac{\lambda \cos k \sin ^{2} k \sin ^{2} \Lambda_{k} t}{\Lambda_{k}^{2}}+\frac{1}{4}, \\
\left\langle c_{1}(t) c_{2}(t)\right\rangle= & \frac{1}{4 \pi} \int_{-\pi}^{\pi} d k \sin 2 k A(k) B(k)+\frac{1}{8 \pi} \int_{-\pi}^{\pi} d k\left[B(k)^{2}-A(k)^{2}\right](1-\cos 2 k) \\
= & \frac{1}{2 \pi} \int_{0}^{\pi} d k \sin ^{2} k\left[\frac{i \sin 2 \Lambda_{k} t}{\Lambda_{k}}-\cos 2 \Lambda_{k} t-2 \frac{\lambda \sin ^{2} \Lambda_{k} t}{\Lambda_{k}^{2}}(\cos k+\lambda)\right]
\end{aligned}
$$

Note that $\left\langle c_{1}^{\dagger}(t) c_{2}(t)\right\rangle$ is indeed real, so that we have $\rho_{23}^{12}(t)=\rho_{32}^{12}(t)$.

For bipartite entanglement, a commonly used measure for arbitrary states of two qubits is the so-called concurrence [12]. The concurrence is defined as

$$
\begin{aligned}
C(t) & =\max \left\{0,2 \lambda_{\max }(\mathrm{t})-\operatorname{tr} \sqrt{\rho^{12}(\mathrm{t}) \tilde{\rho}^{12}(\mathrm{t})}\right\}, \\
\tilde{\rho}^{12}(t) & =\sigma_{y} \otimes \sigma_{y} \rho^{12 *}(t) \sigma_{y} \otimes \sigma_{y},
\end{aligned}
$$

where $\lambda_{\max }$ is the largest eigenvalue of the matrix $\sqrt{\rho^{12}(t) \tilde{\rho}^{12}(t)}$.

In the present case,

$$
\rho^{12}(t)=\left(\begin{array}{cccc}
\rho_{11}^{12}(t) & 0 & 0 & \rho_{14}^{12}(t) \\
0 & \rho_{22}^{12}(t) & \rho_{23}^{12}(t) & 0 \\
0 & \rho_{23}^{12}(t) & \rho_{22}^{12}(t) & 0 \\
\rho_{14}^{12 *}(t) & 0 & 0 & \rho_{44}^{12}(t)
\end{array}\right),
$$




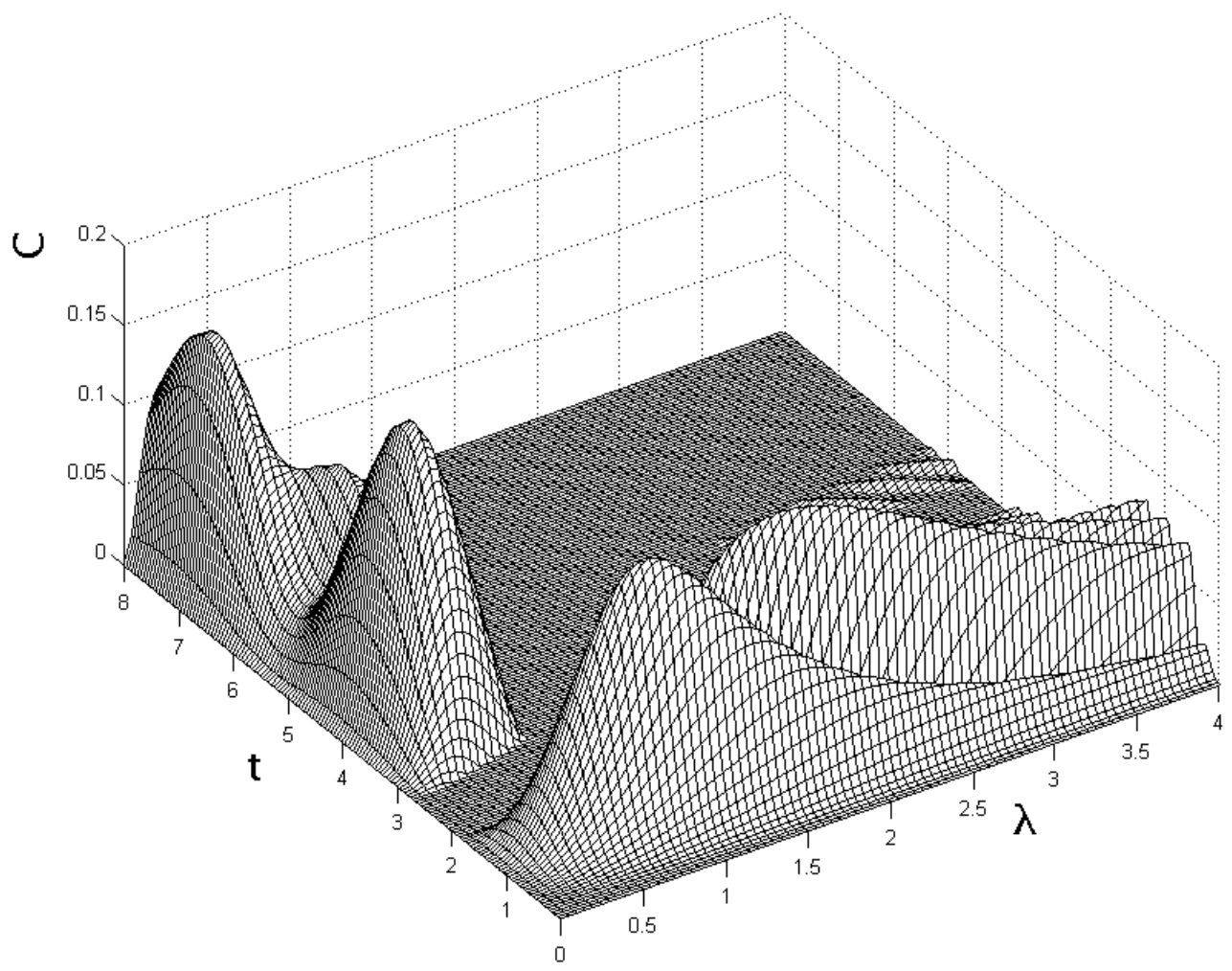

Figure 1: The evolution of bipartite entanglement for the initial state being the "thermal ground state" of the Ising model.

the four eigenvalues of $\sqrt{\rho^{12}(t) \tilde{\rho}^{12}(t)}$ are of the form

$$
\begin{aligned}
& \lambda_{1}(t)=|| \rho_{14}^{12}(t)\left|+\sqrt{\rho_{11}^{12}(t) \rho_{44}^{12}(t)}\right|, \\
& \lambda_{2}(t)=|| \rho_{14}^{12}(t)\left|-\sqrt{\rho_{11}^{12}(t) \rho_{44}^{12}(t)}\right|, \\
& \lambda_{3}(t)=\left|\rho_{22}^{12}(t)+\rho_{23}^{12}(t)\right|, \\
& \lambda_{4}(t)=\left|\rho_{22}^{12}(t)-\rho_{23}^{12}(t)\right| .
\end{aligned}
$$

In Fig.1, we give a plot of the evolution of entanglement as a function of time $t$ and parameter $\lambda$. At the beginning, the reduced density matrix is

$$
\rho^{12}(0)=\frac{1}{4}\left(\begin{array}{cccc}
1 & 0 & 0 & 1 \\
0 & 1 & 1 & 0 \\
0 & 1 & 1 & 0 \\
1 & 0 & 0 & 1
\end{array}\right) .
$$

So that the concurrence equals zero. At any later fixed instant, the entanglement as a function of $\lambda$ may have different behaviors in different time intervals. For times before 


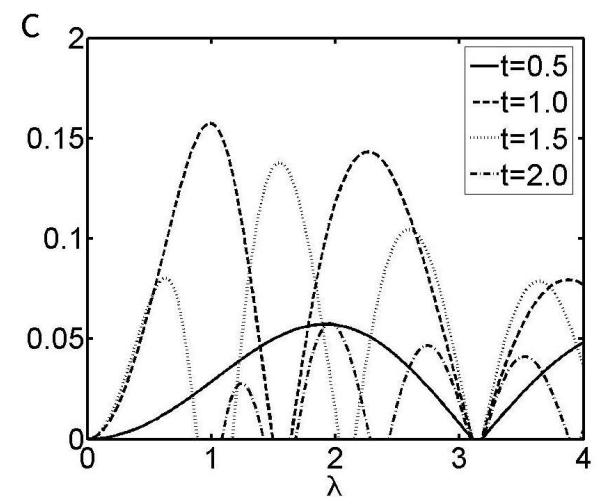

(a)

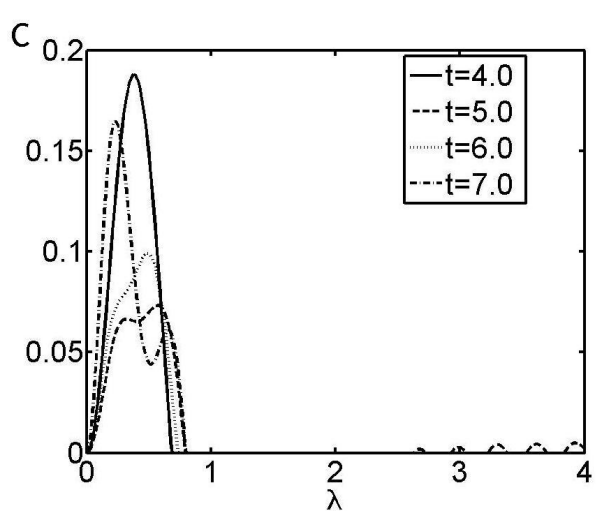

(b)
Figure 2: (a): The concurrence at different times $t=0.5,1.0,1.5,2.0$ as a function of $\lambda$, in the region of $t<2.5$, (b): The concurrence at different times $t=4.0,5.0,6.0,7.0$ as a function of $\lambda$, in the region of $t>3.0$.

about $t=2.5$ (Fig.2 (a)), the entanglement maintains its magnitude for large $\lambda$ with slow oscillations. As $\lambda$ increases, the height of each peak deceases gradually, and finally vanishes as $\lambda \rightarrow \infty$. This is easy to understand. For $\lambda \rightarrow \infty$, the Hamiltonian reduces to the pure Ising model, and the initial state is just the eigenstate of the Ising model. Thus, only a phase factor contributes to the times evolving state, which does not change the entanglement. Physically, this corresponds to $\lambda_{1}=\lambda_{2}=\infty$, hence no quench takes place. For the narrow band $2.47<t<2.69$, there is no entanglement at all for all $\lambda$. For times greater than around $t=3.0$ (Fig.2 (b)), the entanglement emerges abruptly, but only has non-vanishing values for very short interval of $\lambda$. As the time increases, the generations of entanglement are different from each other for different $\lambda$. For $\lambda$ is less than about $\lambda=0.8$ (Fig.3 (a)), the concurrence will encounter more than one maximums. In a time region between the first two maximums, no entanglement appear. Although weak oscillations emerge after this region, the entanglement can last for a fairly long time with a not too small magnitude. For $0.80<\lambda<1.07$, there is only one maximum as the time increases. After a short time, the entanglement vanishes. For $\lambda>1$ (Fig. 3 (b)), strong oscillations emerge. The entanglement reaches its maximum fast, then suddenly decreases to zero. Such a behavior will repeat again at later times, and finally the entanglement vanishes completely.

For any $\lambda$, there is a time $T_{m}(\lambda)$ at which the entanglement reaches its first maximum (Fig.4). This time is a monotonically decreasing function of $\lambda$. Taking its derivative with respect to $\lambda$, we find that the derivative $\frac{d T_{m}(\lambda)}{d \lambda}$ has a minimum at the critical point $\lambda=1$. This interesting property maybe an indicator of the quantum phase transition at the critical point. 


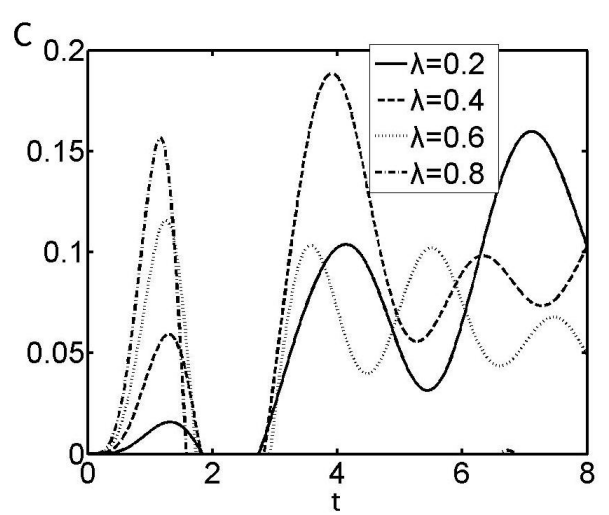

(a)

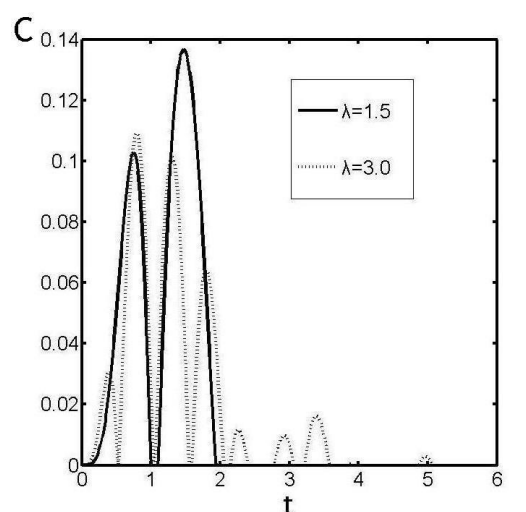

(b)
Figure 3: (a): The dynamics of entanglement for different reciprocal fields $\lambda=0.2,0.4,0.6,0.8$, in the region of $\lambda<0.8$, (b): The dynamics of entanglement for different reciprocal fields $\lambda=1.5,3.0$, in the region of $\lambda>1.07$.

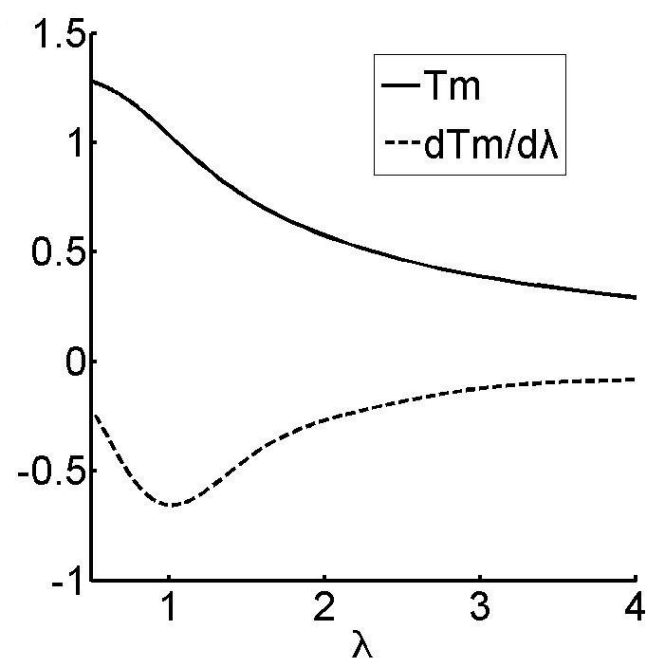

Figure 4: The time at which the entanglement reaches its first maximum and its derivative. 


\section{Brief discussions and conclusions}

In this paper, we have studied the dynamics of the transverse Ising chain prepared in the "thermal ground state" of the pure Ising model. For different values of the reciprocal field, the evolution of the entanglement has dramatic distinctions. We also obtained the times at which the entanglement reaches its first maximum, which is a monotonically decreasing function of the parameter $\lambda$. The derivative of this quantity with respect to the reciprocal field gets its minimum at the critical field. This is a new indicator of the quantum phase transition. Let us finally remark that the initial state we choose is simple to be prepared experimentally. One can first cool the pure Ising system down to near absolute zero, then turn on some desired transverse field at $t=0$ to generate the wanted entanglement.

\section{Acknowledgments}

We would like to thank X. Li for useful discussions. The work was supported partially by the NSF of China under Grant No. 10875129.

\section{References}

[1] M. A. Nielsen and I. L. Chuang, Quantum Computation and Quantum Information (Cambridage University Press, Cambridge, 2000).

[2] R. Horodecki, P. Horodecki, M. Horodecki, and K. Horodecki, Rev. Mod. Phys. 81, 865 (2009).

[3] L. Amico, R. Fazio, A. Osterloh, and V. Vedral, Rev. Mod. Phys. 80, 517 (2008).

[4] T. J. Osborne and M. A. Nielsen, Phys, Rev. A 66, 032110 (2002).

[5] A. Osterloh, L. Amico, G. Falci, and R. Fazio, Nature (London) 416, 608 (2002).

[6] L. Amico, A. Osterloh, F. Plastina, R. Fazio, and G. M. Palma, Phys, Rev. A 69, $022304(2004)$.

[7] A. Sen(De), U. Sen, and M. Lewenstein, Phys, Rev. A 72, 052319 (2005).

[8] H. Wichterich and S. Bose, Phys, Rev. A 79, 060302(R) (2009).

[9] D. Jaksch, C. Bruder, J. I. Cirac, C. W. Gardiner, and P. Zoller, Phys, Rev. Lett. 81, 3108 (1998).

[10] O. Mandel, M. Greiner, A. Widera, T. Rom, T. W. Hansch, and I. Bloch, Nature (London) 425, 937 (2003).

[11] E. Lieb, T. Schultz, and D. Mattis, Ann. Phys. (N.Y.) 16, 407 (1961).

[12] W. K. Wootters, Phys, Rev. Lett. 80, 2245. 